\title{
Contesto biologico e implicazioni etiche della malattia nel Timeo di Platone
}

\author{
Biological Context and Ethics Implications of the Illness in \\ Plato's Timaeus
}

BARBARA BOTTER*

\begin{abstract}
A partire dall'analisi di alcuni passi del Timeo, il testo propone lo studio delle cause e dei tipi di infermità che affliggono la salute umana al fine di mostrare che la maniera in cui il filosofo concepisce le malattie è una chiave per approfondire l'antropologia e l'etica dell'ultimo Platone. Presupposto della ricerca è la visione olistica che caratterizza il Timeo, in cui la condizione umana influenza il livello socio-politico e il livello cosmico, i quali, a loro volta, condizionano e sono condizionati dalla presenza dell'uomo. Per finalizzare lo studio abbiamo circoscritto l'analisi ai passi 69c-90c del Timeo.

Parole chiave: Platone; Etica; Cosmologia; Politica; Malattia; Vizio.
\end{abstract}

\begin{abstract}
Starting with the analysis of some Timaeus passages, the text proposes the study of the causes and of the types of infirmity that afflict human health in order to demonstrate that the way the philosopher conceives illnesses is a key to get a deeper knowledge of the anthropology and ethics of the last Plato. Premise of the research is the holistic vision that characterizes the Timaeus, in which the human condition affects the socio political level and the cosmic level which, in their turn, affect and are affected by the human presence. To finalize the study, we analyzed the pages $69 \mathrm{c}-90 \mathrm{c}$ of the Timaeus.
\end{abstract}

Keywords: Plato; Ethics; Cosmology; Politics; Infirmity; Vice.

\section{Introduzione}

Le differenti maniere attraverso le quali i gruppi sociali nel corso delle epoche guardano e reagiscono alla infermità riflettono non solo le loro convinzioni relative a ciò che è considerato malattia, ma anche una teoria relativa alla costituzione umana, i valori etici e, in generale, una certa visione del mondo. Come chiarisce Van der Ejik, "Essa variedade já começa no plano da experiência pessoal: aquilo que indivíduos ou grupos, em uma sociedade

Recibido: 23/08/2018. Aceptado: 16/12/2018.

* Professore Aggiunto del Dipartimento di Filosofia da Università Federale dello Espírito Santo (UFES), Vitória, Brasil. Indirizzo e mail: barbarabotter@gmail.com. Area di recerca e specializzazione: Storia della Filosofia Antica, in particolare Platone e Aristotele, in relazione alle dottrine etiche, politiche e ontologiche. Principali pubblicazioni: B. Botter, La Creazione di Immagini in Platone. Milano: AlboVersorio 2016. B. Botter, "The Influence of the "honeyed Muse" over the Soul in Plato's Republic". In Boeri, M.; Kanayama, Y.; Mittelmann, J. (org.), Soul and Mind in Greek Thought. Psychological Issues in Plato and Aristotle. New York: Springer 2018, 43-67. B. Botter, Aristotele e i suoi dei. Milano: Carocci 2011. 
determinada, experimentam como dor, enfermidade, deficiência ou desconforto pode variar de um caso para o outro" (Van der Ejik, 2009, 11-12).

È fondamentale per il nostro studio analizzare come Platone comprende, nomina e classifica ciò che egli concepisce come malattia, al fine di gettare luce sull'antropologia platonica e le sue conseguenze sociali e politiche.

Sebbene alcuni esegeti, come il citato Van der Ejik, ritengano che Platone sia il filosofo che differenziò le "malattie dell'anima" dalle "malattie del corpo" e che ciò sia un necessario riflesso della sua concezione dualista, noi difenderemo l'idea che il pensatore mantiene, nel corso del Timeo, una visione integrata dell'uomo e un approccio olistico nella relazione fra individuo, città e cosmo.

Mantenendoci fedeli a tale interpretazione, estranea a qualsiasi visione antropocentrica, nel testo a seguire ci proponiamo di esaminare le seguenti questioni: a) In che misura l'uomo è responsabile per le sue proprie condizioni di limitazione fisica e per le malattie che possono affliggerlo. b) In che modo lo stato di infermità fisico - psichica è relazionato ad una nozione di "infermità etica", ossia qual è la relazione fra vizio e malattia e quali sono le ricadute a livello etico e politico.

\section{Destino del cosmo e dell'uomo}

Nel Timeo Platone descrive dapprima l'orizzonte all'interno del quale pone la riflessione antropologica: il cosmo. I protagonisti, Timeo e Crizia, discutono nel Prologo le ragioni per le quali è necessario dapprima descrivere la costituzione del cosmo in vista della descrizione della costituzione umana e del giusto ordinamento della città. Gli dei ausiliari formano il corpo dell'uomo prendendo a prestito dalla totalità cosmica porzioni di aria, acqua, terra e fuoco, le quali saranno poi restituite al tutto: “ ... e ricevuto il principio immortale del vivente mortale, imitando il loro demiurgo, presero in prestito dal cosmo (apò tô̂ kósmou daneizómenoi $)^{1}$ porzioni di fuoco, di terra, di acqua, di aria, che poi avrebbero dovuto restituirgli (hos apodothēsómena pálin) (T.2 43a1-2. Traduzione di F. Fronterotta; si veda anche 53a-c e 81a). L'idea che per conoscere se stesso l'uomo deve dapprima comprendere l'universo risale ad Anassimandro, cui fa eco Eraclito. La prima parola della filosofia ammonisce l'uomo a non dimenticare che:

Ciò da cui proviene la generazione delle cose che sono, peraltro, è ciò verso cui si sviluppa anche la rovina, secondo necessità: le cose che sono, infatti, pagano l'una all'altra la pena e l'espiazione dell'ingiustizia, secondo l'ordine del tempo. (Anassimandro [in Simplicio], fr. 12 B 1).

La nascita è l'insorgere dell'ingiustizia e questa ingiustizia è pagata con la morte. Eraclito riconosce che la natura è normativa e l'uomo deve agire in accordo con le sue Leggi: "tutte le Leggi umane sono alimentate da una Legge divina" (22B114 DK).

1 Nella traslitterazione del testo greco ci serviamo delle norme di traslitterazione fornite da Fernández Galiano.

2 Per citare i dialoghi platonici useremo seguenti abbreviazioni: T. Timeo; R. Repubblica; L. Leggi; Prot. Protagora; Phil. Filebo; Phaedr. Fedro; Phaed. Fedone; Pol. Politico; Gorg. Gorgia; Cra. Cratilo. 
Il cosmo è il rappresentante dell'ordine all'interno del disordine dominato dalla "Causa Errante" (planōménē aitía, T. 48a), ossia dalla Necessità (anágkēe, T. 47e). L'ordine dell'universo è istituito dall'intervento della causa finale, la quale dapprima dota l'universo di vita (T. 30a-c), quindi contribuisce all'unità e alla direzione di esso (Trindade Santos, 2007, 3) 3 . L'uomo è uno dei molteplici elementi della natura viva e come il cosmo è l'immagine necessariamente imperfetta del paradigma ideale, l'uomo è l'immagine necessariamente imperfetta del cosmo nella sua costituzione fisica, animica e, probabilmente, nel suo destino ${ }^{4}$.

Il fatto che l'esistenza umana sia limitata da un ininterrotto alternarsi di corruzioni e generazioni è giustificato da due ordini di fattori:

1) Da ragioni di ordine fisiologico. Frias ritiene che il filosofo greco si ispirò alle dottrine mediche di Alcmeone di Crotone, secondo il quale gli uomini muoiono perché sono incapaci di unire l'inizio e il fine (fr. 2 apud Frias, 1997, 58-59). Diversamente dai cicli cosmologici, che si realizzano continuamente, i cicli fisiologici della respirazione e della nutrizione (T. 79b-e e 80-81) sono transitori (Mugler, 1958, 42-50).

2) Da ragioni di ordine escatologico. "É preciso assegurar a reprodução e conservação deste sinal material que é o corpo", e la riproduzione "é o meio não só de criar um novo corpo, mas também de transmitir uma alma correspondente a este corpo"5 (Brisson, 2011, 137).

\section{La costituzione elementare del cosmo e la sue ripercussioni nella formazione mate- riale dell'uomo}

Tale quale il cosmo, l'uomo è costituito, al livello fisico, degli stessi triangoli elementari che compongono tutte le cose e che rappresentano, in combinazione matematica, gli elementi di cui tutti gli enti sono costituiti, sebbene nell'uomo gli elementi cosmici non siano presenti allo stato puro e nella pienezza della loro potenza (Phil. 29a).

Tanto il cosmo quando l'uomo sono strutturati in modo matematicamente proporzionato da una iniziale porzione di fuoco e terra a cui il Demiurgo ha aggiunto acqua e aria nella seguente proporzione: fuoco $:$ aria $=$ aria $:$ acqua $=$ acqua $:$ terra in rispetto alla legge di formazione dei solidi (Migliori, 2003). Ciò che discrimina l'uomo rispetto all'universo è che, differentemente dall'universo, nell'uomo il legame fra i triangoli non comporta la stessa stabilità che nel cosmo (T. 32c1-4; cf. 32d1-33a e 33a6-b1; 37e-38a $)^{6}$. Infatti, le radici dei triangoli che lo compongono sono fissate solo con chiodi (desmô̂s) invisibili per la loro piccolezza (T. 43a2-3).

I processi di declino della struttura umana sono naturali e dovuti all'azione della necessità, se provocati dall'invecchiamento e dal tempo, a causa degli effetti della divisione (diákrisis, T. 61d7) e dell'incisione (tomē, T. 61d7, 80e3) degli elementi interni ed esterni al corpo. Il deterioramento è innaturale e precoce se provocato dalle malattie frutto del disordine delle abitudini alimentari, delle condizioni ambientali e del comportamento vizioso (T. 81d-86a).

3 Come già detto nel Fedro 246b-d.

4 Su questo punto le opinioni degli interpreti sono divise. Si veda Robinson, 2007; Berti, 1997; Reale, 2002; Brisson, 2001; Silverman, 2002; Migliori, 2003 e Carone, 2008, 97.

5 Si veda Cra. 399e; Scrivani 2007, 379-402.

6 Si veda Brisson, 2001, in particolare 273, nota 714 . 


\subsection{Invecchiamento e morte $(T .81 b-d)$}

L'invecchiamento è descritto da Timeo come un indebolimento delle radici dei triangoli che compongono gli elementi corporei. Questo tipo di degradazione è inteso da Platone come una conseguenza della lotta (agōn, T. 81c7) fra gli elementi interni ed esterni al corpo. I ripetuti attacchi reciproci fra gli elementi provocano la divisione (temnō, T. 57a2; tomē 80d3; diákrisis 61d7) dei triangoli che costituiscono le radici degli elementi. Gli elementi che hanno maggiore capacità di tagliare interagiscono con i triangoli più deboli, i quali subiscono l'azione dei primi. L'invecchiamento consiste nel progressivo cedimento e smantellamento (chalaō, T. 81c7) delle radici dei triangoli corporei da parte degli elementi i cui triangoli sono più giovani e taglienti $(T .81 \mathrm{~b} 3-81 \mathrm{e} 1)$.

È evidente che l'assunzione degli elementi provenienti dall'esterno attraverso i cicli della respirazione e della nutrizione è necesssaria per la sopravvivenza umana, ma la stessa azione è responsabile per la morte dell'uomo. Perciò, l'uomo si consuma attraverso le stesse azioni che lo mantengono in vita. Il verbo phtinō, alla linea $81 \mathrm{~b} 5$, è indicativo della situazione di corruzione naturale dell'uomo che viene immaginata da Platone come un processo di consumazione e di usura dovuti ad un prolungato esercizio (Morais, 2009).

\subsection{Le malattie del corpo (T. $82 a-86 d)$}

Proponiamo il seguente schema esplicativo che riunisce le differenti malattie e le loro cause.

1. Malattie causate dal disequilibrio tra i quattro elementi dovuto all'eccesso, alla mancanza o alla cattiva distribuzione di uno di essi (T. 82a-82b).

2. Una specie di 1 . sono le malattie dovute alla corruzione dei tessuti corporei, in particolare la carne, le ossa e il midollo osseo (T. 82b-84d). La gravità di queste malattie è dovuta al rovesciamento del processo di formazione delle strutture corporee (anápalin he génesis toútōn, T. 82c6) che porta alla formazione di liquidi umorali nocivi alla salute.

3. Chiudono le malattie che dipendono dal ciclo corrotto della respirazione ( $T$. 84d-85a), dai flegmi (T. 85a-b) e dalla bile (T. 85b-86a). In questi casi, ciò che affligge il corpo dipende, in primo luogo, dalle condizioni climatiche (T. 88d; cf. 86a; 84d-85a) 7 . Particolarmente nocivi alla salute umana sono la formazione del flegma e della bile. Nei casi più gravi, il primo penetra fino alla regione encefalica e si mescola con la bile nera, provocando la perturbazione dei circoli conoscitivi ( $T$. 85a). Infine, la bile può alterare il funzionamento del midollo quand'essa, insinuandosi nel sangue, ne brucia le fibre. Lo stadio ultimo di questo processo è la decomposizione dei triangoli elementari e la dissoluzione fra anima e corpo $(T .85 \mathrm{e} 4-7)^{8}$.

7 Per i dettagli si veda Frias, 2005 e Dulce Reis, 2010 alla quale dobbiamo importanti chiarimenti utili per la redazione del nostro testo.

8 Interessanti sono le riflessioni di Morais, 2009a, in particolare 122-134. 


\section{Le malattie del congiunto corpo-anima}

Per indicare le patologie che affliggono il composto psico-fisico (synanphóteron, T. 86b87b; 87c-88b) Platone utilizza l'espressione "malattie dell'anima che si manifestano attraverso la disposizione del corpo" (tà dè perì psychēn dià sōmátos héxin, T. 86b1). Con questa espressione Platone evidenzia che in tali casi la necessità non è interamente e direttamente responsabile per le conseguenze che si manifestano (Dulce Reis, 2010).

Non è pertinente descrivere queste malattie unicamente come "malattie dell'anima", né unicamente come "malattie del corpo" visto che si tratta di patologie che sono scatenate da fattori corporei o da fattori emotivi ma i cui effetti coinvolgono il composto (Dulce Reis, 2010). In generale, la causa principale delle malattie dell'anima che si manifestano attraverso il corpo è il desequilibrio e il conflitto fra le tre differenti fonti motivazionali del noûs, del thymós e dell'epithymetikón. La perdita di armonia fra le parti dell'anima è dovuta al mal funzionamento o alla totale mancanza di funzionamento del noûs. Per questa ragione, esse sono caratterizzate come forme di "assenza di senno" (ánoian), a loro volta distinte in follia (manía) e ignoranza (amathía, T. 86b).

Le cause sono legate tanto alla cattiva disposizione del corpo e alla inadeguata educazione dell'anima (T. 86d7-e3); quanto a discorsi e a regimi politici perversi (T. 87a7-b4) ${ }^{9}$.

Solo nel caso dell'akolasía (T. 86d5), l'intemperanza sessuale, il flusso di sostanze nocive è attribuito all'eccesso della sostanza accumulata nel midollo e alla mancanza di consistenza del tessuto osseo (T. 86d2-7; cf. 86e3-87a7; Dulce Reis, 2010, 115 e Morais 2009a, 133).

Nel passo $87 \mathrm{c}-88 \mathrm{~b}$ Timeo aggiunge una nota finale. La malattia descritta in questo passo è provocata dal disequilibrio tra la forza dell'anima e quella del corpo. Nel caso del predominio del livello razionale, certe investigazioni (mathēseis kaì zetēsēeis) consumano (katékei, T. 88a3) l'anima; se predomina l'irrascibile, l'uomo manifesta eccessiva irritabilità (perithymós) e amore per le dispute (philonikía T. 88a5). Quando, infine prevale il livello appetitivo, l'uomo mostra una eccessiva preoccupazione per la soddisfazione del desiderio di nutrimento (tropēs T. 88b2).

Nel caso di una supremazia del corpo sul congiunto animico, l'uomo mostra difficoltà nell'apprendimento, stupidità, amnesia e, in generale, ignoranza (T. 88a7-b5).

Per godere di salute è necessario l'equilibrio fra il congiunto fisico psichico (Frias, 2005). Infatti, sebbene l'anima sia considerata più nobile del corpo essa non deve avere una educazione privilegiata e non deve essere eccessivamente nutrita rispetto al corpo (Carone, 2008). Per non incorrere in una guerra civica (stásis) "Non si muova l'anima senza il corpo, né il corpo senza l'anima, in modo tale che ciascuno dei due sia preservato, equilibrato e sano" (T. 88b5-c1. Traduzione nostra).

Questa appendice alla descrizione delle patologie ci è parsa rilevante perché ha gettato chiarezza sull'antropologia vigente nel Timeo. A nostro avviso, il filosofo fa leva su una relazione fra anima e corpo tale da non distinguere l'uno e l'altro al punto da rendere il loro

9 Platone lega chiaramente "una cattiva disposizione del corpo e (kai) una educazione inadeguata" alla pagina 86d7 del Timeo, a cui si aggiungono fattori esterni di ordine sociale e politico (T. 87a7-b4). Si veda Dulce Reis 2009, 107-122; 2010, 111; Frias 2001, 116; Stell 2001, 127); Stalley 1996, 365-366 e 367; Brisson 2015, 454; Cornford 1948, 346. 
rapporto conflittuale, né da fonderli fino a renderli indistinguibili. Utilizzando un ricorso grammaticale tipico del dialetto greco attico, ci sembra che si possa qualificare la relazione fra anima e corpo una relazione "duale". L'azione del "duale" in greco non è accompagnata da un verbo singolare né da un verbo plurale. Anima e corpo non sono divisi e posti l'uno di fronte all'altro, né fusi in una unità che non permetterebbe alcun tipo di relazione. L'anima e il corpo sono legati l'uno all'altro, questo "altro" non essendo, però, "uno tra gli altri". Come afferma Monique Dixsaut,

“L'âme n'est donc pas pour Platon une substance séparée du corps, et si elle doit s'en séparer, sa separation est la conquête d'une pensée que le corps n'alourdit plus mais où elle est déjà présente". Del resto "partout où il y a mouvement et vie il y a aussi âme et une sorte de pénsée et il y a jusque dans le corps, une sagesse" (Dixsaut, 2015, rispettivamente 57 e 58).

\section{Patologia fisico psichica e responsabilità}

È evidente da quanto detto che se l'universo e gli astri sono immortali e autosufficienti, visto che il corpo di cui sono dotati è "amico" (phílon T. 34b7-8) e non produce alcun tipo di necessità (T. 33a-d), e la loro anima deborda di saggezza (T.34b-c; 36e), lo stesso non si può dire degli esseri umani.

La vita umana nasce con l'unione di corpo e anima, ed è esattamente nel momento di questo contatto che il caos ha inizio. Infatti, a causa degli influssi e degli efflussi corporei, i periodi, altrimenti perfetti, dell'anima immortale vengono sconvolti (T. 43a6-b5).

I movimenti violenti, provocati dall'introduzione del nutrimento $(T$. 43b5-6) e dalle sensazioni (T. 43b6-c7), interferiscono con la regolarità dei movimenti psichici. Dopo un certo periodo la situazione si stabilizza (T. 44b4-7), ma, come fa notare Brisson, "même si cette phase critique est maintenant terminée, il ne faut pas croire que tout va pour le mieux" (Brisson, 2015, 430). Se ciò che È buono è bello $(T .87 \mathrm{c} 4-5)$ e ciò che è bello è armonico (T. 87c4; cf. 86d7-e2), la salute è raggiunta attraverso lo sforzo di mantenere l'equilibrio fisico, psichico e psico-fisico. "Et cela en tenant compte du lien de proportionalité qui les lie, c'est-à-dire, en définitive, en assurant la prédominance de l'espèce immortelle de l'âme" (Brisson, 2015, 431).

In questo modo, Platone traccia il programma che deve essere realizzato nel corso della vita umana; un programma che sfida le capacità umane, perché, diversamente dall'universo, la proporzione e l'omeostasi non sono concesse all'uomo nel momento della generazione e in modo definitivo. Esse possono essere messe in questione e facilmente perdute. La rottura della proporzione è la radice e la fonte di ogni errore, vizio e di ogni malattia (Dulce Reis, 2010, 121). Il male (kakía) e la malattia (nósos) hanno, per Platone, una comune origine e sono indissolubilmente legate ( $T$. $86 \mathrm{~d}$-e). Il male fisico e morale consiste nella perdita dell'equilibrio, mentre la salute e la virtù sono legate alla giusta proporzione tra corpo e anima e tra i livelli animici (T. 43e8-44a7; 44a3; 86b2-4). Nella ideale condizione di equilibrio l'azione umana è diretta dalle rotazioni razionali del circolo dell'Identico (T. 42c4-d1), le quali coincidono con il regno del bene, dell'eccellenza e del bello (T. 87c4-5). Questa prospettiva, già presente nel libro IV della Repubblica (444d-e), sarà ribadita nel Sofista, 
in cui il modello di bellezza è legato alla giusta misura e il suo contrario alla mancanza di essa (ámetron, Sph. 226d-230d, in particolare 228d), e sigillerà i libri IX e X delle Leggi (in particolare 863e5-864a8).

Nel Timeo il protagonista osserva che l'uomo non è volontariamente malvagio (kakós), perché la mancanza di proporzione fra le componenti è conseguenza della cattiva disposizione fisica dovuta all'azione della necessità (T. 68e-69a) e dell'inadeguata educazione ricevuta nell'infanzia, di cui sono responsabili principalmente i genitori e gli educatori $(T .87 \mathrm{~b})^{10}$.

Se l'uomo non è responsabile, se non in misura limitata, del suo squilibrio psico fisico, e visto che la patologia e il vizio (kakía, T. 86a7) sono le due faccie di un'erma bifronte, in che misura l'uomo deve essere responsabilizzato per l'una e per l'altra?

\section{Ogni vizio è malattia, ma non ogni malattia è vizio}

Il termine "malvagità" (kakía), in sé, non rimanda necessariamente al comportamento umano all'interno della Polis (Robinson, 2007), perciò non deve essere legato immediatamente ad una condizione di perversione morale. In senso generico, il male indica un allontanamento dal modello di armonia nel corpo e eccellenza etica. Come osserva Carone,

Platão está claramente adaptando o ideal homérico da pessoa que é boa-e-bela a sua concepção [...]. No Timeu "virtude" descreve não só um estado da alma, mas também um estado da pessoa toda, incluindo sua interação harmônica com seu corpo (87d). Em contraste, a assimetria entre corpo e alma, e o exercício excessivo de um às expensas da outra, causa doença e vício, e impede alguém de ser justamente chamado belo e bom (87d-88d)". (Carone, 2008, 105).

Il termine kakía può indicare tanto un disequilibrio in senso generico, quanto la perversione morale cosciente e deliberata. Nel Timeo la distinzione fra le due situazioni non sempre, però, risulta evidente. I seguenti passi che relazionano la malvagità alla responsabilità umana non hanno trovato una esegesi unanime tra gli interpreti e meritano di essere letti con attenzione.

E quasi tutto ciò che si definisce come incapacità di dominare i piaceri ed è oggetto di rimprovero, come se si fosse malvagi volontariamente, non è a giusto titolo che viene rimproverato; giacché nessuno è volontariamente malvagio, ma il malvagio diviene tale per una cattiva disposizione del corpo e per la crescita senza educazione e situazioni del genere sono dannose per chiunque e capitano senza volerlo" (T. 86d7e3. Traduzione di F. Fronterotta).

"Inoltre, se a individui così mal costituiti si aggiungono cattive istituzioni e si tengono, in privato e in pubblico, discorsi conformi alla città [...] allora tutti i malvagi fra noi lo diventano per due ragioni, senza volerlo" (T. 87a7-b4. Traduzione di F. Fronterotta).

10 Un accurato esame delle pagine T. 86 sg. si trova in Dulce Reis, 2009; Dulce Reis, 2010, in particolare 121, 159 e nota 236; e Carone, 2008. 
Hardie e Taylor considerano determinista la posizione di Platone in queste pagine del dialogo. L'uomo non è spontaneamente malvagio e, nel caso in cui la sua anima sia corrotta, ciò è dovuto all'azione della necessità responsabile per le imperfezioni della costituzione corporea e al tipo di educazione ricevuta (T. 86e1-2). Secondo Robinson (Robinson, 2007, 148), le interpretazioni di Hardie e Taylor sono, per così dire, miopi, in quanto escludono il contesto generale delle affermazioni platoniche. Lo studioso ritiene che, sebbene in Platone non esista la "libertà" di scelta, il testo manifesta la presenza di una tendenza umana a cui l'uomo può dare assentimento o meno. Perciò, se determinismo esiste, esso è solo potenziale.

Dello stesso avviso, Carone distacca che, pur avendo ricevuto una educazione inadeguata ed essendo costituito in modo necessariamente imperfetto, l'individuo può rimediare, per quanto gli è possibile (hópêi tis dýnatai). Se il suo comportamento rivela uno stato di perversione morale, ciò significa che egli non ha prodotto nessuno sforzo per correggere le sue carenze costitutive, perciò è in parte responsabile di ciò che è divenuto.

Mesmo esse elemento de necessidade, que é capaz, como tal de impedir nossa atividade racional se negligenciada ou tornada objeto de abuso, pode, inversamente, ser usado para facilitá-la. Tomemos o caso de nosso desejo de alimento [...]. Seria insensato viver uma vida que girasse em torno da satisfação desse tipo de desejo [...]. Por outro lado, tal desejo é natural (cf. 88b1-2) e cumpre um papel teleológico. Assim deveríamos satisfazê-lo não só com o objetivo de atender a uma necessidade fisiológica (70d7-8), mas também no interesse de cultivar o equilíbrio apropriado entre corpo e alma, sem o qual a virtude seria impossível (87c-d)" (Carone, 2008, 97).

Da parte nostra, crediamo che prima di assumere una posizione esegetica di fronte a queste pagine sia decisivo capire a) se e fino a che punto il corpo, nel quale in maggior misura si manifesta l'azione della necessità, ostacola l'azione teleologica del noûs al punto da non permettergli di operare; b) se il corpo è dotato di capacità autonoma di azione.

Relativamente ad a), ci pare che si debba essere cauti ad attribuire unicamente al corpo l'inferiorità umana. È vero, come osserva Robinson (2007, in particolare 139), che la necessità non può essere interamente addomesticata alla ragione e che la sua resistenza è evidente specialmente nella costituzione fisiologica (T. 73a-74b; cf. T. 75a7-b1; cf. 73a-74b).

Ma il fatto che parte dei movimenti corporei siano gestiti dalle forze erranti, non rende l'uomo una vittima innocente della necessità e non basta ad esimerlo della propria responsabilità in relazione alla sua condizione psico fisica e alle ripercussioni di essa in ambito etico e cosmico.

La salute umana, includendo in questa espressione non solo un equilibrio fisico ma allo stesso tempo etico, risulta da una combinazione di fattori fisici, educativi, psicologici e ambientali combinati in giuste proporzioni (T. 70a-b e 71b-c). L'anima razionale è responsabile per il mantenimento e il ristabilimento dell'armonia fisica e per la moderazione degli eccessi psichici del thymós e dell'epithymetikón in modo analogo all'azione della causa finale in relazione all'azione della necessità pre-cosmica (Carone, 2008).

Per rispondere $\mathrm{a}$ b), ossia determinare se per Platone il corpo abbia potenzialità autonoma di azione, accompagnamo alcune riflessioni nel Fedone, sebbene questo dialogo non 
appartenga probabilmente alla stessa fase della redazione del Timeo ${ }^{11}$, dialogo tardivo, ma appartenga al gruppo di scritti attribuiti alla maturità del filosofo. Seguendo il raziocinio di Socrate, il corpo non è la reale causa delle proprie scelte. Da ciò non segue, troppo sbrigativamente, che il corpo è semplicemente un carcere dell'anima. La struttura fisica può essere trasformata da ostacolo a strumento, da fonte potenziale di disordine a mezzo (óchema) di cui la ragione si serve per attingere i propri fini (T. 44d-e; 69c). Il corpo fornisce all'uomo la possibilità di azione (Phaed. 79b1-2), perciò, l'azione umana dipende dal modo in cui l'anima si relaziona al corpo. In altri termini, è questa relazione che fa del corpo un nemico o un alleato, una fonte di vizio o di azione corretta (Borges de Araujo Jr., 2009, 95-96).

Stando così le cose, l'uomo non è esente di responsabilità per la sua costituzione antropologica e per la sua condizione etica.

\section{Determinismo vs responsabilità}

Il Timeo presenta una specie di sfida in relazione al concetto di responsabilità. Infatti, da un lato il protagonista ripete che il demiurgo è senza colpa (anaitios, T. 42d; e3; cf. 37c4-7); dall'altro lato, si ritrova nel Timeo la nota affermazione che nessuno è malvagio volontariamente (kakòs mèn gàr hékōn oudeís, T. 86d7-e1). Siamo coscienti del fatto che la relazione fra l'uomo e il demiurgo non può essere presa in seria considerazione, visto che i due enti sono incommensurabili, tuttavia, ci pare necessario distaccare il fatto che Platone insiste nel mettere in evidenza la bontà del demiurgo e la sua estraneità in relazione alle azioni umane:

Il demiurgo, lo si è più volte ricordato, è ‘buono' (29a) e non può perciò volere né compiere il male. Radicalizzando questo concetto si comprende quanto Timeo precisa qui: l'artefice non vuole neanche rischiare di essere corresponsabile di eventuali, involontarie malvagità, o piuttosto errori, che le sue creature (in questo caso le anime) potrebbero in futuro commettere. Questa posizione, di totale deresponsabilizzazione della divinità prima e suprema, va sottolineata con forza". (Fronterotta 2015, 233 nota 161).

Il famoso "paradosso socratico", secondo il quale nessuno agisce male di buon grado, è un refrain nei dialoghi platonici. Infatti, appare nell'Apologia (25e-26a), nel Protagora (345d-e; 358c-d), nel Gorgia (467c-468c; 509e); nel Menone (77b-78b), nella Repubblica (589c), nel Sofista (230a), e nelle Leggi (V 731c, 734b, e IX 860d). Di fatto, come dice lo stesso termine, si tratta di un paradosso (para - doxa) nella misura in cui si scontra con l'opinione comune espressa da personaggi come Glaucone e Trasimaco nella Repubblica, Polo e Callicle nel Gorgia, secondo i quali solo il malvagio è vizioso e, in quanto tale, felice, mentre, chi rispetta la giustizia agisce solo per obbligo legale o per mantenere una certa credibilità pubblica. Dal punto di vista socratico, non si tratta affatto di un paradosso, ma della conseguenza di principi etici e antropologici. Come rileva Tremblay (2014, 253-254), si tratta, in particolare, dei seguenti postulati: a) ogni desiderio è direzionato al bene che gli è proprio; b) il proprio bene è essenzialmente lo stesso che il bello (tò kalón), il quale non

11 Sulle ipotesi relative alla datazione dei dialoghi seguiamo le suggestioni di Trindade Santos 2005, 182 nota 2. 
differisce dal bene morale (tò agathón $)^{12}$. Da questi due postulati segue necessariamente che nessuno desidera e acconsente al suo proprio male ${ }^{13}$. L'errore della doxa è un errore conoscitivo, dovuto alla mancanza della scienza di ciò che è bene, confuso con la sua ombra, ossia ricchezza, onore, reputazione, bellezza (Apol. 29d-e). Il paradosso è scatenato dall'inabilità della doxa di passare dal primo al secondo postulato, ossia dal fatto che l'uomo sia per natura incline alla ricerca del proprio bene e che quest'ultimo non differisca dal bene morale. Secondo Platone, solo la conoscenza del bene permette all'uomo la sua realizzazione. Infatti,

[...] le désir seul ne suffit pas à atteindre son objet, qu'il ne contient pas em lui-même les ressorts indispensables à la réalisation de son penchant, ce dernier fût-il tout à fait naturel. Autrement dit, la tension désirante vers le bien moral ne suffit guère à garantir l'exactitude de son orientation. Encore faut-il que le desir trouve à eclairer son chemin par l'éclat de quelque source lumineuse extérieure, et cet éclat ne saurait provenir d'autre chose pour Socrate que de la connaissance elle -même, véritable phare de notre inclination morale" (Tremblay 2014, 257).

Ora, la conoscenza di cui l'intellettualismo socratico si fa portavoce non è intesa come una scienza intellettuale e esterna all'individuo, visto che, in tal caso, la conoscenza verbale del bene, sarebbe condizione necessaria e sufficiente della realizzazione di esso. Ciò che giustifica l'affermazione paradossale "nessuno agisce male volontariamente" è il fatto che la sophía di cui parla Platone è la sophía che agisce dall'interno dell'anima umana e che si identifica con la piena consapevolezza dei fini naturali dell'esistenza umana, ossia con la phrónesis (Dixsaut 2015) ${ }^{14}$.

Tout ce que nous appelons 'vertu' n'en est vraiment que si de la pensée l'accompagne, mais 'accompagner' ne signifie pas 's'ajuter'. Vertu et pensée ne sont pas des entités closes dont chacune pourrait être définie isolément, ce sont des termes si intérieurs l'un à l'autre que la pensée traverse toutes les vertus et pénètre chacune d'elles sans en être une partie ni occuper une place dans sa définition. [...] Il est tout autant impossible que la pensée sage ne modifie pas l'âme qui en est capable, et elle n'a pas pour effet de constituer un sujet savant mais de rendre l'âme totalement vertueuse. L'intelligence prend le nom de phronesis, losqu'il s'agit de dire son effet en l'âme" (Dixsaut 2015, 266).

Tradizionalmente gli esegeti utilizzano i termini "volontariamente" e "involontariamente" per rendere hékon e ákon, sebbene non esista una vera e propria facoltà della "volontà" in Platone, come fanno notare Dulce Reis (2010) e gli studi più recenti di Brisson (2015) e Dixsaut (2015). Secondo Dulce Reis, alle pagine 86d7-e3, Platone sta negando che un uomo possa essere malvagio senza che altri fattori siano determinanti per questa condizione. La studiosa pensa all'uomo nel Timeo come ad un "agente moral livre", manifestando il suo

12 Si veda al proposito l'argomento edonista nel Protagora 358a-e, e le sue conseguenze nel passo 359c5-360d6 e Capra 2001, in particolare l'analisi dedicata ai passi citati.

13 Si veda a proposito Tremblay 2014, 254 e Merker 2006, 195.

14 Su questo punto si veda anche Tremblay 2014. 
consenso con Robinson a proposito di una mancanza di "determinismo somático no diálogo" (Dulce Reis, 2010, 113) ${ }^{15}$.

Brisson (2015), ribadisce che Platone non conosce il concetto di "libertà" nella comune accezione del termine, ma riconosce negli scritti del filosofo greco la presenza dell'idea di una azione autonoma (hékon), così come dei concetti di decisione (aíresis) e di desiderio (boúlēsis).

Jamais on ne trouve l'expression d'une volonté dont le choix, fait em pleine liberté, implique soit le salut ou la perte de l'âme. Pour Platon, il est impossible de souhaiter autre chose que le bien qui s'impose à la raison. Or, la raison peut être troublée. Elle est alors sujette à l'erreur" (Brisson, 2015, 451-452).

Monique Dixsaut (2015) conferma l'assenza della facoltà volitiva in Platone. Il termine stesso non trova corrispettivo nella lingua greca. "Ce que l'on traduit "volontaire" se dit ékon, "de bon gré" et signifie l'obéissance à un ordre que n'est pas ressenti comme une contrainte". Perciò, "agir de plein gré” significa dare il proprio assentimento, lasciarsi comandare, ma non da una forza esterna all'anima, essendo l'anima "le thêatre d'affrontemets entre forces désirantes, forces dont l'existence est d'ailleurs révélée par leurs conflits" (Dixsaut, 2015, rispettivamente 256 e 258). Autori come Monte Johnson (2014) e Pierre Destrée (2014) hanno rilevato la presenza di alcune questioni che sono strettamente relazionate con la nozione di responsabilità morale e con il concetto, essenzialmente aristotelico, di éph'hēmîn, ciò che dipende da noi, già in autori come Democrito e Platone. Pur non ignorando la presenza di questi elementi, crediamo che la nozione di responsabilità morale appaia solo con Aristotele e non ancora come un termine tecnico nella teoria dell'azione (D. Frede 2014), né come supporto di una teoria indeterministica dell'azione (Bobzien 2014). Da parte nostra, ammettiamo senza difficoltà la presenza di concetti che evocano la capacità decisionale e di autonomia dell'agire in Platone. Solo neghiamo che questi concetti abbiano la portata morale strictu sensu, tale quale essi acquisteranno in epoche successive. In particolare, riteniamo che se una nozione di volontà possa essere attribuita a Platone, essa non possa essere intesa come un facoltà psichica autonoma, fonte di autodeterminazione assoluta nella condotta delle azioni umane (Tremblay 2014) ${ }^{16}$. In assenza di tale facoltà, "tout le système conceptuel impliqué dans notre reprèsentation du volontaire se trouve mis en cause dans le contexte grec" (Vernant 2001, p. 47), "mais aussi subsidiairement toute notre compréhension de la pénalité se voit, au contact dépaysant de Platon, radicalement confondue" (Tremblay 2014, 253).

15 Secondo Stalley, visto che le cause che spingono l'uomo ad agire in un certo modo sono i cattivi discorsi e i regimi politici corrotti (T. 87b4), le affermazioni del filosofo sono un ammonimento a prendersi cura della propria anima (Stalley, 1996, 368).

16 Interessante a questo proposito il contributo di M. Frede, A Free Will: Origins of the Notion in Ancient Thought. Non utilizziamo direttamente questo contributo nel nostro testo, in quanto l'autore non realizza una esegesi dei testi antichi, bensì presenta essenzialmente il risultato delle proprie riflessioni sui testi. Frede non prende posizione sulla questione se si possa, o meglio, se sia necessario abolire la nozione di volontà libera, bensì si schiera a favore e contro alcuni concetti filosofici associati a questa nozione. 
L'individuo è dotato di tre facoltà psichiche: il noûs e i livelli animici inferiori ${ }^{17}$. Questi ultimi, sebbene in sé siano moralmente neutri, ricevono una tonalità etica dal loro rapporto con la parte dirigente dell'anima. Quando l'anima razionale perde la sua funzione dirigente ed è passivamente trasportata dai livelli psichici inferiori non attinge il giudizio retto e l'uomo è soggetto all'errore, alla malattia e al vizio (T. 42b2). Questo è il livello in cui l'uomo deve essere responsabilizzato. Osserva Brisson,

La culpabilité de l'homme ne peut équivaloir à l'inauguration d'une chaîne causale dans le champ de la morale, mais à une négligence par rapport à la proportion des éléments de son être. [...] Ce n'est pas parce qu'il a décidé de l'être qu'il est, mais, au contrarie, parce qu'il n'a pas decide de ne pas l'être" (Brisson, 2015, 454).

Platone aggiunge, tuttavia, delle attenuanti.

Di fatto, esistono alcuni fattori che sfuggono al dominio del soggetto. L'uomo, infatti, è il prodotto della società in cui vive e le pratiche educative (ginnastica, musica e filosofia) sono pratiche eminentemente sociali $(T .87 \mathrm{~b})$. Perciò, il problema della corruzione morale e fisica dell'uomo ricade, almeno in parte, nella città, perché se la città è retta da un regime politico corrotto, essa diviene corrotta e produce uomini corrotti (R.471sg.). Ciononostante, come osserva Carone, Platone non priva educandi e generati delle loro responsabilità. Infatti,

embora possa ser verdadeiro que ninguém age errado voluntariamente no sentido de que alguém não faria o mal ou seria mau caso tivesse a apropriada educação e o correto equilíbrio psicossomático (86d-e) [...] ainda se é responsável pelas próprias ações e escolhas" (Carone, 2008, 97).

Nel libro X delle Leggi Platone sembra addirittura ampliare la responsabilità umana ad un orizzonte cosmico. Alla pagina 900e il filosofo considera la malattia e la pestilenza come casi di errore (hamártēma) e di insaziabilità (pleonexía) umane, utilizzando termini di accentuato senso etico (L. 906a-b). L'idea rientra perfettamente nella visione olistica di Platone, secondo la quale il cosmo è un organismo dotato di vita (T. 34a-40d; cf. Phaedr. $245 \mathrm{c}-\mathrm{d})$ e l'uomo è parte costituente del "Tutto a causa del quale egli esiste e non viceversa" (L. 903c7-d1).

La visione delle Leggi, condivisa nella Repubblica e nel Timeo, suggerisce chiaramente che ciò che importa è l'eccellenza della totalità, la parte esistendo in vista della totalità e non vice-versa ${ }^{18}$, ogni sua alterazione ripercuotendosi nelle altre e nella totalità $(R .462 \mathrm{c}-\mathrm{d})^{19}$.

17 Pur distinguendo tre livelli animici, siamo persuasi che la nozione di anima non deve essere pensata come un congiunto di parti. Del resto, pensare al termine "parti" in un senso letterale all'interno di una concezione dell'anima come ente immateriale sembra quasi una contraddizione in termini. Su questo punto si veda Dulce Reis, 2009.

18 Alle pagine 30c-d, 39e-40a e 41a-c, Timeo osserva che gli enti dotati di vita, quindi l'uomo incluso, devono esistere in vista della preservazione della totalità. Perciò, la vita umana si rivela un elemento imprescindibile per la vita dell'organismo cosmico ed è la vita (bíos) del cosmo il fine in vista del quale ogni parte è generata.

19 Su questo risvolto della filosofia platonica, si veda Carone, 2008, 275-277. 
Se l'uomo non è necessariamente determinato, bensí parzialmente responsabile per le scelte relative alla propria salute e interamente responsabile per le sue azioni, in quali casi le sue decisioni e i suoi atti sono soggetti all'attenzione della Legge?

\section{Malattia, malvagità e responsabilità penale}

Dato lo stretto legame tra ingiustizia (adikía) e malattia (cf. T. 43e-44a; 86b; 86d7-87c; $R .444 \mathrm{~d}-\mathrm{e}$; Sph. 226d-230d), per sapere se e, in che misura, un uomo, la cui anima è malata, è malvagio e politicamente colpevole, è necessario ricorrere all'esame di alcuni passi del libro IX delle Leggi, i quali pongono i requisiti che determinano quando una persona è passibile di condanna.

Una prima distinzione deve essere operata fra azioni ingiuste realizzate intenzionalmente (hékon) e azioni ingiuste non intenzionali (ákon) (L. 860e6-861a6). La distinzione ci induce a credere che il filosofo non attribuisca alle azioni realizzate non intenzionalmente lo stesso peso che alle altre forme di ingiustizia. Ciononostante, come fa notare Dulce Reis, non è l'intenzionalità dell'azione che determina se essa è ingiusta ( $L$. 861d4-7), né la presenza di un danno, sebbene tali componenti non possano essere ignorati dal legislatore (Dulce Reis, 2010, 153). Nel passo 861e-862c il filosofo discrimina gli elementi che determinano il carattere ingiusto di un'azione.

L'analisi del passo rivela che: a) non è il danno o il beneficio conseguenti una azione che ne rivelano il carattere giusto o ingiusto; b) non esistono due specie di ingiustizia, le forme intenzionali e non intenzionali; c) solo se una azione è provocata da una persona di carattere (éthos) ingiusto e il cui modo di agire (trópos) è ingiusto, essa è una forma di ingiustizia in senso proprio; d) una azione che provoca danno, ma è realizzata da un individuo il cui abito è giusto non può essere considerata una forma di ingiustizia (Dulce Reis, 2010) ${ }^{20}$.

Risulta chiaro che una anima ingiusta è una anima malata, ma non necessariamente malvagia, come era evidente dall'analisi del passo 86b-88b del Timeo. Ciò che determina la qualità etica di un atto, ossia rivela se una anima malata è anche ingiusta, sono l'éthos, ossia il carattere, e il trópos, il suo modo di agire. Come spiega Dulce Reis ${ }^{21}$,

O que evidencia ser má uma alma injusta não é, então, o estado injusto de sua alma, mas o seu modo de agir, isto é, o fato de se tratar de uma ação consentidamente má, deliberadamente prejudicial a outrem. Portanto, um homem é mau (assim como seu ato) se o seu caráter for injusto e o seu modo de agir também o for" (Dulce Reis, 2010, 158).

La famosa affermazione socratica secondo la quale nessuno è malvagio volontariamente non trova conferma nelle Leggi, dalle cui pagine si avvince che l'individuo malvagio è tale intenzionalmente (cf. L. 862c6-8). Se si seguono le conseguenze dell'intellettualismo socratico, è necessario concludere che è impossibile per l'individuo desiderare e commentere

20 Siamo debitori alle analisi di Dulce Reis, 2010 per la comprensione di questo difficile passo delle Leggi. Si vedano, in particolare, le pagine 149-160.

21 Per l'esegesi dei passi delle Leggi e le distinzioni fra azioni ingiuste, malvagie, azioni intenzionali e danno siamo debitori e interamente grati a Dulce Reis, 2010, 146-169. 
azioni ingiuste, se non per incoscienza del bene, visto che il male solo può essere il frutto di un desiderio intriso di ignoranza (Tremblay 2014).

Secondo il raziocinio dell'ultimo scritto platonico, chi possiede un carattere malvagio e agisce in modo ingiusto pratica il male intenzionalmente, visto che lo stato mentale dell'agente è la molla dell'azione. Al contrario, chi provoca danno, ma non agisce intenzionalmente, non può essere considerato malvagio e il suo atto deve essere considerato frutto di un'anima malata, dannoso ma non criminale. Un atto volontario incline al male può essere considerato un atto criminale, visto che l'uomo è, per lo meno in parte, responsabile per la sua condizione fisica e morale. L'uomo può controllare l'omeostasi fisiologica attraverso una alimentazione adeguata e non eccessiva, la pratica ginnica e musicale (T. 72d-e); e la formazione di un éthos giusto attraverso l'interesse per gli studi intellettuali (T. 88a-89a) che assicurano il predominio del ciclo dell'Identico nella conduzione retta del corpo e dei livelli animici inferiori.

Infine, non avrebbe senso la considerazione mostrata da Platone per le terapie mediche e politiche se l'uomo non avesse la possibilità di modificare la propria attitudine e fosse determinato da condizioni che indipendono da lui. Nel passo 862c6-863a2 delle Leggi il filosofo stabilisce che uno dei compiti del legislatore è di prescrivere una cura per le anime malate, visto che l'ingiustizia è una malattia psichica (en psycheî nóson, L. 862c6). Dall'esame del Timeo è emerso che un'anima è malata quando i tre differenti livelli animici sono in conflitto (stásin) fra loro e il germe del conflitto è scatenato dalla deviazione dei movimenti concentrici dell'anima razionale causata dalla corruzione dei circoli dell'Identico e del Diverso, di cui l'uomo è responsabile $(T .87 \mathrm{c})$.

\section{Conclusione}

In questo contributo ci siamo proposti di analizzare il legame che Platone stabilisce fra la malattia fisica e il vizio al fine di ponderare la percentuale di responsabilità che gli deve essere attribuita di fronte a se stesso, alla città e alla natura.

Partendo dall'analisi dei limiti umani dal punto di vista fisico e dalla descrizione delle malattie che possono affliggere l'uomo, abbiamo evidenziato che la causa principale delle malattie è il disequilibrio fra anima e corpo, e il conflitto fra le tre differenti fonti motivazionali del noûs, del thymós e dell'epithymetikón. La rottura della proporzione è la radice e la fonte di ogni malattia e vizio. Tuttavia, l'omeostasi nell'uomo non è stabilita nel momento della generazione e in modo definitivo. È compito dell'individuo riprodurre e mantenere nel microcosmo la mediazione operata dalla causa finale a livello macrocosmico.

La responsabilità umana non è limitata all'atteggiamento che l'uomo manifesta in relazione al proprio benessere psico fisico, ma comporta anche un versante etico. Nel Timeo la connessione tra la malattia e il vizio è esplicita $(T .86 \mathrm{~d}-\mathrm{e})$ e ciò conferma quanto già chiarito nel Protagora. La stretta relazione fra malattia e vizio, salute e virtù, è imprescindibile per Platone, visto che la virtù, in generale sinonimo di giustizia, non è oggetto di scelta, bensì costituisce l'érgon propriamente umano differenziandolo dagli animali. L'uomo non ha giustizia, bensì è giusto secondo natura. La ricerca sulla virtù della giustizia mette in gioco se stessi, e non può essere ridotta ad un oggetto di scelta né può muovere da ipotesi soggettive. Quando l'anima razionale perde la sua funzione dirigente ed è passivamente trasportata 
dagli altri livelli psichici non attinge il giudizio retto e l'uomo è soggetto alla patologia e alla malvagità. Di ciò l'uomo è responsabile. Se il comportamento umano rivela uno stato di perversione morale, ciò significa che egli non ha prodotto nessuno sforzo per correggere le proprie limitazioni costitutive.

Ciononostante, la responsabilità in senso ampio non comporta necessariamente un obbligo penale. Dall'esame di alcuni passi del libro IX delle Leggi è emerso che un'anima ingiusta è una anima malata, ma non necessariamente malvagia. Solo chi possiede un carattere (éthos) malvagio e agisce in modo (trópos) ingiusto pratica il male intenzionalmente; mentre chi provoca un danno, ma non agisce intenzionalmente, non deve essere condannato e il suo atto è conseguenza di un'anima malata, ma non criminale.

\section{Bibliografia}

\section{Testi antichi e traduzioni}

Platone, (1992, 20013), Timeu/Crítias. Trad. Com. L. Brisson. Paris: Flammarion, 1992.

Platone, (2015), Timeo. Trad. F. Fronterotta. Milano: BUR.

Platone, (1999), As Leis. Trad. Com. E. Bini. Bauru, São Paulo: EDIPRO.

\section{Commentatori}

Bobzien, S. (2014), “Aristotle's Nicomachean Ethics 1113b7-8 and free choices”. In M. Zingano, R. Salles, P. Destrée (org.), What is up to us? Studies on Agency and Responsibility in Ancient Philosophy. Sankt Augustin: Academia Verlag 2014, 59-74.

Borges de Araujo Jr., A. (2009), "Sócrates, o corpo, a morte e a tarefa do pensamento: um estudo do Fédon de Platão". In M. Campolina Diniz Peixoto, A saúde dos Antigos. São Paulo: Loyola 2009, 95-105.

Brisson, L. (1997), "Perception sensible et raison dans le Timée". Interpreting the Timaeus/ Critias. Proceedings of the IV Symposium Platonicum (congrès). Sankt-Augustin: Academia Verlag 1997, 307-316.

Brisson, L. (1998, 2015), Le Même et l'Autre dans la structure ontologique du Timée de Platon. Sankt-Augustin: Academia Verlag.

Brisson, L. (2011), "O continuum da vida", in L. Brisson e F. Fronterotta (org.), Platão: Leituras. São Paulo: Loyola 2011, 133-141.

Capra, A. (2001), Agon Logon. Il Protagora di Platone fra eristica e commedia. Milano: LED.

Carone, G. (2008), A cosmologia de Platão e suas dimensões éticas. São Paulo: Loyola.

Cornford, F.M. (1948), Plato's Cosmology. 2. Ed. London: Routledge and Kegan Paul.

Destrée, P. (2014), "How can our fate be up to us? Plato on the myth of Er". ". In M. Zingano, R. Salles, P. Destrée (org.), What is up to us? Studies on Agency and Responsibility in Ancient Philosophy. Sankt Augustin: Academia Verlag 2014, 25-38.

Dixsaut, M. (2015), Platon - Nietzsche. L'autre manière de philosopher. Paris: Fayard. 
Dulce Reis, M. (2009), "A relação entre a teoria da tripartição da alma e a teoria éticopolítica platônica”. In M. Campolina Diniz Peixoto (org.), A saúde dos antigos. São Paulo: Loyola 2009, 107-123.

Dulce Reis, M. (2010) Virtude e Vício. Tripartição e Unidade da Alma no Timeu e nas Leis de Platão. Rio de Janeiro: 7Letras FAPEMIG.

Ejik van der, Ph. (2009), "Os conceitos de saúde mental na medicina e na filosofia gregas dos séculos V e IV a.C.”. In M. Campolina Diniz Peixoto (org.), A saúde dos antigos. São Paulo: Loyola 2009, 11-23.

Frede, D. (2014), "Free Will in Aristotle". In M. Zingano, R. Salles, P. Destrée (org.), What is up to us? Studies on Agency and Responsibility in Ancient Philosophy. Sankt Augustin: Academia Verlag 2014, 29-58.

Frias, I. (1997), Platão leitor de Hipocrates, Monografia de Mestrado. Universidade: PUCRio.

Frias, I. (2001), “A relação corpo-alma no Timeu em função do binômio saúde-doença". Cadernos de Atas da ANPOF. Rio de Janeiro: 1, 2001, 111-116.

Frias, I. (2005), Doença do corpo, doença da alma: medicina e filosofia na Grécia Clássica. São Paulo: Loyola.

Migliori, M. (2003), "Il problema della generazione nel Timeo". In C. Natali, e St. Maso, Plato Physicus. Amsterdam: Hakkert 2003, 97-120.

Johnson, M. R. (2014), "Changing our minds: Democritus on what is up to us". In M. Zingano, R. Salles, P. Destrée (org.), What is up to us? Studies on Agency and Responsibility in Ancient Philosophy. Sankt Augustin: Academia Verlag 2014, 7-24.

Merker, A. (2006), “Nul n'est méchant de son plein gré”. In Brisson, L. e Fronterotta F. (org.), Lire Platon. Paris: PUF, 189-203.

Morais, K. L. F. (2009), A Unidade corpo-alma na fisiologia-ética do Timeu de Platão. Tese (Mestrado). Universidade Federal de Minas Gerais.

Morais, K. L. F. (2009a), “A relação alma-corpo no Timeu de Platão". In M. Campolina Diniz Peixoto (org.), A saúde dos antigos. São Paulo: Loyola 2009, 123-135.

Mugler, CH. (1958), "Alcmeon et les cycles physiologiques de Platon". Revue des Études Grecques, LXXI, p. 42-50.

Reale, G. (2002), Corpo, alma e saúde: o conceito de homem de Homero a Platão. São Paulo: Paulus.

Robinson, T.M. (2007), A Psicologia de Platão. São Paulo: Loyola.

Scrivani, F. 2997), "Spunti e riflessioni in margine alla concezione platonica della riproduzione umana", in L. Napolitano (org.), La sapienza di Timeo. Riflessioni in margine al Timeo di Platone. Milano: Vita e Pensiero 2007, 379-402.

Silverman, A. (2002), The Dialectic of Essence. Princeton.

Stalley, R.F. (1996), "Punishment and the physiology of the Timaeus". Classical Quarterly. 46, 257-370.

Steel, C. (2001), “The Moral Purpose of the Human Body. A Reading of Timaues 69-72”. Phronesis XLVI, 2, 105-128.

Tremblay, U. G. (2014), "Nul ne fait le mal de son plein gré: remarques sue les implications pénales du paradoxe socratique". Phares. Vol 14, hiver 2014, 251-269. 
Trindade Santos, J. T. S. (2005), "Districando il Cratilo". In Casertano, G. (org.), Il Cratilo di Platone. Strutture e Problematiche. Napoli: Loffredo 2005, 182-201.

Trindade Santos, J. T. S. (2007), "A alma no Timeu”. Eikasia. Revista de filosofia, 12 Extraordinario 1, 97A-112A.

Vernant, P. (2001), "Ébauche de la volonté dans la tragédie grecque". In Mythe et Tragédie em Grèce Ancienne. Vol. I. Paris: La Découverte 2001. 
